\title{
Properties of Solution Set of Tensor Complementarity Problem
}

\author{
Yisheng Song* Gaohang $\mathrm{Yu}^{\dagger}$
}

\begin{abstract}
The tensor complementarity problem is a specially structured nonlinear complementarity problem, then it has its particular and nice properties other than ones of the classical nonlinear complementarity problem. In this paper, it is proved that a tensor is an S-tensor if and only if the tensor complementarity problem is feasible, and each Q-tensor is an S-tensor. Furthermore, the boundedness of solution set of the tensor complementarity problem is equivalent to the uniqueness of solution for such a problem with zero vector. For the tensor complementarity problem with a strictly semi-positive tensor, we proved the global upper bounds for solution of such a problem. In particular, the upper bounds keep in close contact with the smallest Pareto $H-(Z-)$ eigenvalue.
\end{abstract}

Key words: Tensor complementarity, S-tensor, strictly semi-positive, feasible vector.

AMS subject classifications (2010): 47H15, 47H12, 34B10, 47A52, 47J10, 47H09, 15A48, 47H07.

\section{Introduction}

The nonlinear complementarity problem was introduced by Cottle in his Ph.D. thesis in 1964. In the last decades, many mathematical workers have concentrated a lot of their en-

*Corresponding author. School of Mathematics and Information Science and Henan Engineering Laboratory for Big Data Statistical Analysis and Optimal Control, Henan Normal University, XinXiang HeNan, P.R. China, 453007. Email: songyisheng1@gmail.com. The work was supported by the National Natural Science Foundation of P.R. China (Grant No. 11571905), Program for Innovative Research Team (in Science and Technology) in University of Henan Province(14IRTSTHN023).

†School of Mathematics and Computer Sciences, Gannan Normal University, Ganzhou, 341000, China. Email: maghyu@163.com. This author's work was supported by the National Natural Science Foundation of P.R. China (Grant No. 10926029,11001960), NCET Programm of the Ministry of Education (NCET 13-0738), science and technology programm of Jiangxi Education Committee (LDJH12088). 
ergy and attention on this classical problem because of a multitude of interesting connections to numerous disciplines and a wide range of important applications in operational research, applied science and technology such as optimization, economic equilibrium problems, contact mechanics problems, structural mechanics problem, nonlinear obstacle problems, traffic equilibrium problems, and discrete-time optimal control. For more detail, see [1, 2, 3, 4] and references therein. Well over a thousand articles and several books have been published on this classical subject, which has developed into a well-established and fruitful discipline in the field of mathematical programming.

The linear complementarity problem is a special case of nonlinear complementarity problem. It is well-known that the linear complementarity problem has wide and important applications in engineering and economics (Cottle, Pang and Stone [5] and Han, Xiu and Qi [3]). Cottle and Dantzig [6] studied the the existence of solution of the linear complementarity problem with the help of the structure of the matrix. Some relationship between the unique and existence of solution of the linear complementarity problem and semi-monotoncity of the matrices were showed by Eaves [7], Karamardian [8], Pang [9, 10] and Gowda [11, respectively. Cottle [12] studied some classes of the complete Q-matrix (a matrix is called the complete Q-matrix iff it and all its principal sub-matrices are Q-matrix), and obtained that each completely Q-matrix is a strictly semi-monotone matrix.

The tensor complementarity problem, as a special type of nonlinear com- plementarity problems, is a new topic emerged from the tensor community, inspired by the growing research on structured tensors. At the same time, the tensor complementarity problem, as a natural extension of the linear complementarity problem seems to have similar properties to the problem, and to have its particular and nice properties other than ones of the classical linear complementarity problem. So how to obtain the nice properties and their applications of the tensor complementarity problem will be very interesting by means of the special structure of higher order tensor (hypermatrix).

The notion of the tensor complementarity problem is used firstly by Song and Qi [13], and they showed the existence of solution for such a problem with some classes of structured tensors. In particular, they showed that the nonnegative tensor complementarity problem has a solution if and only if all principal diagonal entries of such a tensor are positive. Che, Qi, Wei [14] showed the existence of solution for the symmetric positive definite tensor complementarity problem and copositive tensors. Luo, Qi, Xiu [15] studied the sparsest solutions to $Z$-tensor complementarity problems. Song and Qi [16] studied the solution of the semi-positive tensor complementarity problem, and obtained that a symmetric tensor is (strictly) semi-positive if and only if it is (strictly) copositive.

In this paper, we will study the properties of solution of the tensor complementarity problem by means of the special structure of tensors. Namely, it will be proved that the solution set of the tensor complementarity problem is bounded if and only if such a tensor 
is a $\mathrm{R}_{0}$-tensor. We will present the global upper bounds for solution of the tensor complementarity problem with a strictly semi-positive tensor.

In section 2, we will give some notions, definitions and basic facts, which will be used in the context. In section 3, we will introduce the concept of S-tensor and give its two equivalent definitions by means of the tensor complementarity problem. Subsequently, it is proved that each Q-tensor is an S-tensor, and a tensor is an S-tensor if and only if the corresponding tensor complementarity problem is feasible. Furthermore, the solution set of the tensor complementarity problem is bounded if and only if such a problem with zero vector has a unique solution. Finally, with the help of the smallest Pareto $H-(Z-)$ eigenvalue, we will discuss the global upper bounds for solution of the tensor complementarity problem with a strictly semi-positive tensor.

\section{Preliminaries}

Throughout this paper, we use small letters $x, y, v, \alpha, \cdots$, for scalars, small bold letters $\mathbf{x}, \mathbf{y}, \cdots$, for vectors, capital letters $A, B, \cdots$, for matrices, calligraphic letters $\mathcal{A}, \mathcal{B}, \cdots$, for tensors. All the tensors discussed in this paper are real. Let $I_{n}:=\{1,2, \cdots, n\}$, and $\mathbb{R}^{n}:=\left\{\left(x_{1}, x_{2}, \cdots, x_{n}\right)^{\top} ; x_{i} \in \mathbb{R}, i \in I_{n}\right\}, \mathbb{R}_{+}^{n}:=\left\{x \in \mathbb{R}^{n} ; x \geq \mathbf{0}\right\}, \mathbb{R}_{-}^{n}:=\left\{\mathbf{x} \in \mathbb{R}^{n} ; x \leq \mathbf{0}\right\}$, $\mathbb{R}_{++}^{n}:=\left\{\mathbf{x} \in \mathbb{R}^{n} ; x>\mathbf{0}\right\}$, where $\mathbb{R}$ is the set of real numbers, $\mathbf{x}^{\top}$ is the transposition of a vector $\mathbf{x}$, and $\mathbf{x} \geq \mathbf{0}(\mathbf{x}>\mathbf{0})$ means $x_{i} \geq 0\left(x_{i}>0\right)$ for all $i \in I_{n}$.

Let $F$ be a nonlinear function from $\mathbb{R}^{n}$ into itself. The nonlinear complementarity problem is to find a vector $\mathbf{x} \in \mathbb{R}^{n}$ such that

$$
\mathbf{x} \geq \mathbf{0}, F(\mathbf{x}) \geq \mathbf{0}, \text { and } \mathbf{x}^{\top} F(\mathbf{x})=0,
$$

or to show that no such vector exists. Let $A=\left(a_{i j}\right)$ be an $n \times n$ real matrix and $F(\mathbf{x})=$ $\mathbf{q}+A \mathbf{x}$. Then such a nonlinear complementarity problem is called the linear complementarity problem, denoted by $\operatorname{LCP}(A, \mathbf{q})$, i.e., to find $\mathbf{x} \in \mathbb{R}^{n}$ such that

$$
\mathbf{x} \geq \mathbf{0}, \mathbf{q}+A \mathbf{x} \geq \mathbf{0}, \text { and } \mathbf{x}^{\top}(\mathbf{q}+A \mathbf{x})=0
$$

or to show that no such vector exists.

In 2005, Qi [17] introduced the concept of positive (semi-)definite symmetric tensors. A real $m$ th order $n$-dimensional tensor (hypermatrix) $\mathcal{A}=\left(a_{i_{1} \cdots i_{m}}\right)$ is a multi-array of real entries $a_{i_{1} \cdots i_{m}}$, where $i_{j} \in I_{n}$ for $j \in I_{m}$. Denote the set of all real $m$ th order $n$-dimensional tensors by $T_{m, n}$. Then $T_{m, n}$ is a linear space of dimension $n^{m}$. Let $\mathcal{A}=\left(a_{i_{1} \cdots i_{m}}\right) \in T_{m, n}$. If the entries $a_{i_{1} \cdots i_{m}}$ are invariant under any permutation of their indices, then $\mathcal{A}$ is called a symmetric tensor. Denote the set of all real $m$ th order $n$-dimensional symmetric tensors by $S_{m, n}$. Then $S_{m, n}$ is a linear subspace of $T_{m, n}$. We denote the zero tensor in $T_{m, n}$ by $\mathcal{O}$. 
Let $\mathcal{A}=\left(a_{i_{1} \cdots i_{m}}\right) \in T_{m, n}$ and $\mathbf{x} \in \mathbb{R}^{n}$. Then $\mathcal{A} \mathbf{x}^{m-1}$ is a vector in $\mathbb{R}^{n}$ with its $i$ th component as

$$
\left(\mathcal{A} \mathbf{x}^{m-1}\right)_{i}:=\sum_{i_{2}, \cdots, i_{m}=1}^{n} a_{i i_{2} \cdots i_{m}} x_{i_{2}} \cdots x_{i_{m}}
$$

for $i \in I_{n}$. Then $\mathcal{A} \mathbf{x}^{m}$ is a homogeneous polynomial of degree $m$, defined by

$$
\mathcal{A} \mathbf{x}^{m}:=\mathbf{x}^{\top}\left(\mathcal{A} \mathbf{x}^{m-1}\right)=\sum_{i_{1}, \cdots, i_{m}=1}^{n} a_{i_{1} \cdots i_{m}} x_{i_{1}} \cdots x_{i_{m}} .
$$

A tensor $\mathcal{A} \in T_{m, n}$ is called positive semi-definite if for any vector $\mathbf{x} \in \mathbb{R}^{n}, \mathcal{A} \mathbf{x}^{m} \geq 0$, and is called positive definite if for any nonzero vector $\mathbf{x} \in \mathbb{R}^{n}$ and an even number $m, \mathcal{A} \mathbf{x}^{m}>0$. Recently, miscellaneous structured tensors are widely studied, for example, Zhang, Qi and Zhou [18] and Ding, Qi and Wei [19] for M-tensors, Song and Qi [20] for P- $\left(\mathrm{P}_{0}\right)$ tensors and B- $\left(\mathrm{B}_{0}\right)$ tensors, Qi and Song [21] for B- $\left(\mathrm{B}_{0}\right)$ tensors, Song and Qi [22] for infinite and finite dimensional Hilbert tensors, Song and Qi 23] for E-eigenvalues of weakly symmetric nonnegative tensors and references therein.

Definition 2.1. Let $\mathcal{A}=\left(a_{i_{1} \cdots i_{m}}\right) \in T_{m, n}$.

(i) The tensor complementarity problem, denoted by $\operatorname{TCP}(\mathcal{A}, \mathbf{q})$, is to find $\mathbf{x} \in \mathbb{R}^{n}$ such that

$$
\begin{aligned}
\mathbf{x} & \geq \mathbf{0} \\
\mathbf{q}+\mathcal{A} \mathbf{x}^{m-1} & \geq \mathbf{0} \\
\mathbf{x}^{\top}\left(\mathbf{q}+\mathcal{A} \mathbf{x}^{m-1}\right) & =0
\end{aligned}
$$

or to show that no such vector exists.

(ii) A vector $\mathbf{x}$ is said to be (strictly) feasible iff $\mathbf{x}$ satisfies the inequality (2.1) and (strict) inequality (2.2).

(iii) The $\operatorname{TCP}(\mathcal{A}, \mathbf{q})$ is said to be (strictly) feasible iff a (strictly) feasible vector exists.

(iv) The set of all feasible vector of the $T C P(\mathcal{A}, \mathbf{q})$ is said to be its feasible region.

(v) The $\operatorname{TCP}(\mathcal{A}, \mathbf{q})$ is said to be solvable iff there is a feasible vector satisfying the equation (2.3).

(vi) $\mathcal{A}$ is called a $\mathbf{Q}$-tensor $([13])$ iff the $\operatorname{TCP}(\mathcal{A}, \mathbf{q})$ is solvable for all $\mathbf{q} \in \mathbb{R}^{n}$.

(vii) $\mathcal{A}$ is called a $\mathbf{R}_{0}$-tensor ([13]) iff the $T C P(\mathcal{A}, \mathbf{0})$ has unique solution.

(viii) $\mathcal{A}$ is called a $\mathbf{R}$-tensor ([13]]) iff it is a $R_{0}$-tensor and the $\operatorname{TCP}(\mathcal{A}, \mathbf{q})$ has unique solution for $\mathbf{q}=(1,1, \cdots, 1)^{\top}$. 
Let $\mathbf{w}=\mathbf{q}+\mathcal{A} \mathbf{x}^{m-1}$. Then a feasible vector $\mathbf{x}$ of the $\operatorname{TCP}(\mathcal{A}, \mathbf{q})$ is its solution if and only if

$$
x_{i} w_{i}=x_{i} q_{i}+x_{i}\left(\mathcal{A} \mathbf{x}^{m-1}\right)_{i}=0 \text { for all } i \in I_{n} .
$$

Recently, Song and Qi [13] extended the concepts of (strictly) semi-monotone matrices to (strictly) semi-positive tensors.

Definition 2.2. Let $\mathcal{A}=\left(a_{i_{1} \cdots i_{m}}\right) \in T_{m, n}$. $\mathcal{A}$ is said to be

(i) semi-positive iff for each $\mathbf{x} \geq 0$ and $\mathbf{x} \neq \mathbf{0}$, there exists an index $k \in I_{n}$ such that

$$
x_{k}>0 \text { and }\left(\mathcal{A} \mathbf{x}^{m-1}\right)_{k} \geq 0 ;
$$

(ii) strictly semi-positive iff for each $\mathbf{x} \geq \mathbf{0}$ and $\mathbf{x} \neq \mathbf{0}$, there exists an index $k \in I_{n}$ such that

$$
x_{k}>0 \text { and }\left(\mathcal{A} \mathbf{x}^{m-1}\right)_{k}>0 ;
$$

(iii) a $\mathbf{P}$-tensor(Song and Qi [20]) iff for each $\mathbf{x}$ in $\mathbb{R}^{n}$ and $\mathbf{x} \neq \mathbf{0}$, there exists $i \in I_{n}$ such that

$$
x_{i}\left(\mathcal{A} \mathbf{x}^{m-1}\right)_{i}>0
$$

(iv) a $\mathbf{P}_{0^{-}}$-tensor (Song and Qi [20]) iff for every $\mathbf{x}$ in $\mathbb{R}^{n}$ and $\mathbf{x} \neq \mathbf{0}$, there exists $i \in I_{n}$ such that $x_{i} \neq 0$ and

$$
x_{i}\left(\mathcal{A} \mathbf{x}^{m-1}\right)_{i} \geq 0 ;
$$

(v) copositive (Qi [24]) if $\mathcal{A} \mathbf{x}^{m} \geq 0$ for all $\mathbf{x} \in \mathbb{R}_{+}^{n}$;

(vi) strictly copositive $(Q i[24])$ if $\mathcal{A} \mathbf{x}^{m}>0$ for all $\mathbf{x} \in \mathbb{R}_{+}^{n} \backslash\{\mathbf{0}\}$.

Lemma 2.1. (Song and Qi [13, Corollary 3.3, Theorem 3.4]) Each strictly semi-positive tensor must be a R-tensor, and each $R$-tensor must be a $Q$-tensor. A semi-positive $R_{0}$-tensor is a Q-tensor.

Song and Qi [25] introduced the concept of Pareto $H-(Z-)$ eigenvalue and used it to portray the (strictly) copositive tensor. The number and computation of Pareto $H-(Z-)$ eigenvalue see Ling, He and Qi [26], Chen, Yang and Ye [27].

Definition 2.3. Let $\mathcal{A}=\left(a_{i_{1} \cdots i_{m}}\right) \in T_{m, n}$. A real number $\mu$ is said to be

(i) Pareto $H$-eigenvalue of $\mathcal{A}$ iff there is a non-zero vector $\mathbf{x} \in \mathbb{R}^{n}$ satisfying

$$
\mathcal{A} \mathbf{x}^{m}=\mu \mathbf{x}^{T} \mathbf{x}^{[m-1]}, \mathcal{A} \mathbf{x}^{m-1}-\mu \mathbf{x}^{[m-1]} \geq 0, \mathbf{x} \geq 0 .
$$


(ii) Pareto $Z$-eigenvalue of $\mathcal{A}$ iff there is a non-zero vector $\mathbf{x} \in \mathbb{R}^{n}$ satisfying

$$
\mathcal{A} \mathbf{x}^{m}=\mu\left(\mathbf{x}^{T} \mathbf{x}\right)^{\frac{m}{2}}, \mathcal{A} \mathbf{x}^{m-1}-\mu\left(\mathbf{x}^{T} \mathbf{x}\right)^{\frac{m}{2}-1} \mathbf{x} \geq 0, \mathbf{x} \geq 0 .
$$

Lemma 2.2. (Song and Qi [25, Theorem 3.1,3.3, Corollary 3.5]) Let $\mathcal{A} \in S_{m, n}$ be strictly copositive. Then

(i) $\mathcal{A}$ has at least one Pareto $H$-eigenvalue $\lambda(\mathcal{A}):=\min _{\substack{\mathbf{x} \geq 0 \\\|\mathbf{x}\| m=1}} \mathcal{A} \mathbf{x}^{m}$ and

$$
\lambda(\mathcal{A})=\min \{\lambda ; \lambda \text { is Pareto } H \text {-eigenvalue of } \mathcal{A}\}>0
$$

(ii) $\mathcal{A}$ has at least one Pareto $Z$-eigenvalue $\mu(\mathcal{A}):=\min _{\substack{\mathbf{x} \geq 0 \\\|\mathbf{x}\|_{2}=1}} \mathcal{A} \mathbf{x}^{m}$ and

$$
\mu(\mathcal{A})=\min \{\mu ; \mu \text { is Pareto } Z \text {-eigenvalue of } \mathcal{A}\}>0 \text {. }
$$

\section{Solution of the $\operatorname{TCP}(\mathcal{A}, \mathbf{q})$}

\subsection{S-tensor and feasible solution of the $\operatorname{TCP}(\mathcal{A}, \mathbf{q})$}

We first introduce the concept of the S-tensor, which is a natural extension of S-matrix [5].

Definition 3.1. Let $\mathcal{A}=\left(a_{i_{1} \cdots i_{m}}\right) \in T_{m, n}$. $\mathcal{A}$ is said to be a

(i) S-tensor iff the system

$$
\mathcal{A} \mathrm{x}^{m-1}>\mathbf{0}, \mathbf{x}>\mathbf{0}
$$

has a solution;

(ii) $\mathbf{S}_{0}$-tensor iff the system

$$
\mathcal{A} \mathbf{x}^{m-1} \geq \mathbf{0}, \mathbf{x} \geq \mathbf{0}, \mathbf{x} \neq \mathbf{0}
$$

has a solution.

Proposition 3.1. Let $\mathcal{A} \in T_{m, n}$. Then $\mathcal{A}$ is a $S$-tensor if and only if the system

$$
\mathcal{A} \mathrm{x}^{m-1}>\mathbf{0}, \mathrm{x} \geq \mathbf{0}
$$

has a solution.

Proof It follows from Definition 3.1 that the necessity is obvious. Now we show the sufficiency. In fact, if there exists $\mathbf{y}$ such that

$$
\mathcal{A} \mathbf{y}^{m-1}>\mathbf{0}, \mathbf{y} \geq \mathbf{0}
$$


clearly, $\mathbf{y} \neq \mathbf{0}$. Since $\mathcal{A} \mathbf{y}^{m-1}$ is continuous on $\mathbf{y}$, it follows that $\mathcal{A}(\mathbf{y}+t \mathbf{e})^{m-1}>0$ for some small enough $t>0$, where $\mathbf{e}=(1,1, \cdots, 1)^{\top}$. It is obvious that $\mathbf{y}+t \mathbf{e}>0$. So $\mathcal{A}$ is an S-tensor.

Now, by means of the solution of the $\operatorname{TCP}(\mathcal{A}, \mathbf{q})$, we may give the following equivalent definition of S-tensor.

Theorem 3.2. Let $\mathcal{A} \in T_{m, n}$. Then $\mathcal{A}$ is a $S$-tensor if and only if the $\operatorname{TCP}(\mathcal{A}, \mathbf{q})$ is feasible for all $\mathbf{q} \in \mathbb{R}^{n}$. Meanwhile, each Q-tensor must be an $S$-tensor.

Proof Let $\mathcal{A}$ is a S-tensor. Then it follows from Definition 3.1 of S-tensor that there exists $\mathbf{y}$ such that

$$
\mathcal{A} \mathbf{y}^{m-1}>\mathbf{0}, \mathbf{y}>\mathbf{0}
$$

So for each $\mathbf{q} \in \mathbb{R}^{n}$, there is $t>0$ such that

$$
\mathcal{A}(\sqrt[m-1]{t} \mathbf{y})^{m-1}=t \mathcal{A} \mathbf{y}^{m-1} \geq-\mathbf{q}
$$

Clearly, $\sqrt[m-1]{t} \mathbf{y}>\mathbf{0}$, and so $\sqrt[m-1]{t} \mathbf{y}$ is a feasible vector of the $\operatorname{TCP}(\mathcal{A}, \mathbf{q})$.

On the other hand, if the $\operatorname{TCP}(\mathcal{A}, \mathbf{q})$ is feasible for all $\mathbf{q} \in \mathbb{R}^{n}$, we take $\mathbf{q}<\mathbf{0}$. Let $\mathbf{z}$ is a feasible solution of the TCP $(\mathcal{A}, \mathbf{q})$. Then

$$
\mathbf{z} \geq \mathbf{0} \text { and } \mathbf{q}+\mathcal{A} \mathbf{z}^{m-1} \geq \mathbf{0}
$$

and hence

$$
\mathcal{A} \mathbf{z}^{m-1} \geq-\mathbf{q}>\mathbf{0} .
$$

So $\mathbf{z}$ is a solution of the system (3.1). It follows from Proposition 3.1 that $\mathcal{A}$ is an $\mathrm{S}$ tensor.

\section{$3.2 \mathbf{R}_{0}$-tensor and boundedness of solution set of the $\operatorname{TCP}(\mathcal{A}, \mathbf{q})$}

Theorem 3.3. Let $\mathcal{A} \in T_{m, n}$. Then the following three conclusions are equivalent:

(i) $\mathcal{A}$ is $R_{0}$-tensor;

(ii) For each $\mathbf{q} \in \mathbb{R}^{n}$ and each $t, s \in \mathbb{R}$ with $t>0$, the set

$$
\Gamma(\mathbf{q}, s, t)=\left\{\mathbf{x} \geq \mathbf{0} ; \mathbf{q}+\mathcal{A} \mathbf{x}^{m-1} \geq \mathbf{0} \text { and } \mathbf{x}^{\top} \mathbf{q}+t \mathcal{A} \mathbf{x}^{m} \leq s\right\}
$$

is bounded;

(iii) For each $\mathbf{q} \in \mathbb{R}^{n}$, the solution set of the $\operatorname{TCP}(\mathcal{A}, \mathbf{q})$ is bounded. 
Proof (i) $\Rightarrow$ (ii). Suppose that there exist $\mathbf{q}^{\prime} \in \mathbb{R}^{n}, s^{\prime} \in \mathbb{R}$ and $t^{\prime}>0$ such that the set $\Gamma\left(\mathbf{q}^{\prime}, s^{\prime}, t^{\prime}\right)$ is not bounded. Let a sequence $\left\{\mathbf{x}^{k}\right\} \subset \Gamma\left(\mathbf{q}^{\prime}, s^{\prime}, t^{\prime}\right)$ be an unbounded sequence.

Then the sequence $\left\{\frac{\mathbf{x}^{k}}{\left\|\mathbf{x}^{k}\right\|}\right\}$ is bounded, and so there exists $\mathbf{x}^{\prime} \in \mathbb{R}^{n}$ and a subsequence $\left\{\frac{\mathbf{x}^{k_{j}}}{\left\|\mathbf{x}^{k_{j}}\right\|}\right\}$ such that

$$
\lim _{j \rightarrow \infty} \frac{\mathbf{x}^{k_{j}}}{\left\|\mathbf{x}^{k_{j}}\right\|}=\mathbf{x}^{\prime} \neq \mathbf{0} \text { and } \lim _{j \rightarrow \infty}\left\|\mathbf{x}^{k_{j}}\right\|=\infty .
$$

From the definition of $\Gamma\left(\mathbf{q}^{\prime}, s^{\prime}, t^{\prime}\right)$, it follows that

$$
\frac{\mathbf{q}^{\prime}}{\left\|\mathbf{x}^{k_{j}}\right\|^{m-1}}+\mathcal{A}\left(\frac{\mathbf{x}^{k_{j}}}{\left\|\mathbf{x}^{k_{j}}\right\|}\right)^{m-1} \geq \mathbf{0} \text { and } \frac{\mathbf{x}^{\top} \mathbf{q}^{\prime}}{\left\|\mathbf{x}^{k_{j}}\right\|^{m}}+t^{\prime} \mathcal{A}\left(\frac{\mathbf{x}^{k_{j}}}{\left\|\mathbf{x}^{k_{j}}\right\|}\right)^{m} \leq \frac{s^{\prime}}{\left\|\mathbf{x}^{k_{j}}\right\|^{m}},
$$

and hence, by the continuity of $\mathcal{A} \mathrm{x}^{m}$ and $\mathcal{A} \mathrm{x}^{m-1}$, let $j \rightarrow \infty$,

$$
\mathcal{A}\left(\mathbf{x}^{\prime}\right)^{m-1} \geq \mathbf{0} \text { and } \mathcal{A}\left(\mathbf{x}^{\prime}\right)^{m} \leq 0 .
$$

Since $\mathbf{x}^{\prime} \geq \mathbf{0}$, we have

$$
\mathcal{A}\left(\mathbf{x}^{\prime}\right)^{m}=\left(\mathbf{x}^{\prime}\right)^{\top} \mathcal{A}\left(\mathbf{x}^{\prime}\right)^{m-1} \geq \mathbf{0} .
$$

Thus, $\mathcal{A}\left(\mathbf{x}^{\prime}\right)^{m}=0$, and hence, $\mathbf{x}^{\prime}$ is a nonzero solution of the $\operatorname{TCP}(\mathcal{A}, \mathbf{0})$. This contradicts the assumption that $\mathcal{A}$ is $\mathrm{R}_{0}$-tensor.

(ii) $\Rightarrow$ (iii). It follows from the definition of $\Gamma(\mathbf{q}, s, t)$ that

$$
\Gamma(\mathbf{q}, 0,1)=\left\{\mathbf{x} \geq \mathbf{0} ; \mathbf{q}+\mathcal{A} \mathbf{x}^{m-1} \geq \mathbf{0} \text { and } \mathbf{x}^{\top}\left(\mathbf{q}+\mathcal{A} \mathbf{x}^{m-1}\right)=0\right\} .
$$

That is, $\Gamma(\mathbf{q}, 0,1)$ is the solution set of the $\operatorname{TCP}(\mathcal{A}, \mathbf{q})$. So the conclusion follows.

(iii) $\Rightarrow$ (i). Suppose $\mathcal{A}$ is not $\mathrm{R}_{0}$-tensor. Then the $\operatorname{TCP}(\mathcal{A}, \mathbf{0})$ has a nonzero solution $\mathbf{x}^{*}$, and so $\mathbf{x}^{*} \in \Gamma(\mathbf{0}, 0,1)$. Since $\mathbf{x}^{*} \neq \mathbf{0}, \tau \mathbf{x}^{*} \in \Gamma(\mathbf{0}, 0,1)$ for all $\tau>0$. Therefore, the set $\Gamma(\mathbf{0}, 0,1)$ is not bounded. This contradicts the assumption (iii). So $\mathcal{A}$ is $\mathrm{R}_{0^{-}}$-tensor.

It is known that each strictly semi-positive tensor is an R-tensor and each $\mathrm{P}$-tensor is a strictly semi-positive tensor (Song and Qi [13]). The following conclusions are obvious.

Corollary 3.4. Let $\mathcal{A}$ be a strictly semi-positive tensor. Then for each $\mathbf{q} \in \mathbb{R}^{n}$, the solution set of the $\operatorname{TCP}(\mathcal{A}, \mathbf{q})$ is bounded.

Corollary 3.5. Let $\mathcal{A}$ be a P-tensor. Then for each $\mathbf{q} \in \mathbb{R}^{n}$, the solution set of the $\operatorname{TCP}(\mathcal{A}, \mathbf{q})$ is bounded.

\subsection{Solution of $\operatorname{TCP}(\mathcal{A}, q)$ with strictly semi-positive tensors}

In this section, we discuss the global upper bound for solution of $\operatorname{TCP}(\mathcal{A}, \mathbf{q})$ with strictly semi-positive and symmetric tensor $\mathcal{A}$. Song and Qi [16] showed the following conclusion about a symmetric tensor. 
Lemma 3.1. (Song and Qi [16, Theorem 3.2, 3.4]) Let $\mathcal{A}=\left(a_{i_{1} \cdots i_{m}}\right) \in T_{m, n}$. Then a symmetric tensor $\mathcal{A}$ is strictly semi-positive if and only if it is strictly copositive. Moreover, the $\operatorname{TCP}(\mathbf{q}, \mathcal{A})$ has a unique solution $\mathbf{0}$ for $\mathbf{q} \geq \mathbf{0}$ when $\mathcal{A}$ is strictly semi-positive.

Theorem 3.6. Let $\mathcal{A}=\left(a_{i_{1} \cdots i_{m}}\right) \in S_{m, n}$ be strictly semi-positive. If $\mathbf{x}$ is a solution of the $\operatorname{TCP}(\mathbf{q}, \mathcal{A})$, then

$$
\|\mathbf{x}\|_{m}^{m-1} \leq \frac{\left\|(-\mathbf{q})_{+}\right\|_{\frac{m}{m-1}}}{\lambda(\mathcal{A})}
$$

where $\lambda(\mathcal{A})$ is defined in Lemma 2.2 (i), $\mathbf{x}_{+}:=\left(\max \left\{x_{1}, 0\right\}, \max \left\{x_{2}, 0\right\}, \cdots, \max \left\{x_{n}, 0\right\}\right)^{\top}$ and $\|\mathbf{x}\|_{m}:=\left(\sum_{i=1}^{n}\left|x_{i}\right|^{m}\right)^{\frac{1}{m}}$.

Proof It follows from Lemma 2.2 and 3.1 that

$$
\lambda(\mathcal{A})=\min \{\lambda ; \lambda \text { is Pareto } H \text {-eigenvalue of } \mathcal{A}\}=\min _{\substack{\mathbf{y} \geq 0 \\\|\mathbf{y}\| m=1}} \mathcal{A} \mathbf{y}^{m}>0 .
$$

Since $\mathrm{x}$ is a solution of the $\operatorname{TCP}(\mathbf{q}, \mathcal{A})$, we have

$$
\mathcal{A} \mathbf{x}^{m}-\mathbf{x}^{\top}(-\mathbf{q})=\mathbf{x}^{\top}\left(\mathcal{A} \mathbf{x}^{m-1}+\mathbf{q}\right)=0, \mathcal{A} \mathbf{x}^{m-1}+\mathbf{q} \geq \mathbf{0} \text { and } \mathbf{x} \geq \mathbf{0} .
$$

Suppose that $\mathbf{q} \geq \mathbf{0}$. Then $\mathbf{x}=\mathbf{0}$ by Lemma 3.1, the conclusion is obvious. So we may assume that $\mathbf{q}$ is not nonnegative, then $\mathbf{x} \neq \mathbf{0}$ (suppose not, $\mathbf{x}=\mathbf{0}, \mathcal{A} \mathbf{x}^{m-1}+\mathbf{q}=\mathbf{q}$, which contradict to the fact that $\mathcal{A} \mathbf{x}^{m-1}+\mathbf{q} \geq \mathbf{0}$ ). Therefore, we have

$$
\frac{\mathcal{A} \mathbf{x}^{m}}{\|\mathbf{x}\|_{m}^{m}}=\mathcal{A}\left(\frac{\mathbf{x}}{\|\mathbf{x}\|_{m}}\right)^{m} \geq \lambda(\mathcal{A})>0 \text {. }
$$

Thus, we have

$$
0<\|\mathbf{x}\|_{m}^{m} \lambda(\mathcal{A}) \leq \mathcal{A} \mathbf{x}^{m}=\mathbf{x}^{\top}(-\mathbf{q}) \leq \mathbf{x}^{\top}(-\mathbf{q})_{+} \leq\|\mathbf{x}\|_{m}\left\|(-\mathbf{q})_{+}\right\|_{\frac{m}{m-1}} .
$$

The desired conclusion follows.

Theorem 3.7. Let $\mathcal{A}=\left(a_{i_{1} \cdots i_{m}}\right) \in S_{m, n}$ be strictly semi-positive. If $\mathbf{x}$ is a solution of the $\operatorname{TCP}(\mathbf{q}, \mathcal{A})$, then

$$
\|\mathbf{x}\|_{2}^{m-1} \leq \frac{\left\|(-\mathbf{q})_{+}\right\|_{2}}{\mu(\mathcal{A})}
$$

where $\mu(\mathcal{A})$ is defined by (2.8) in Lemma 2.2.

Proof It follows from Lemma 2.2 and 3.1 that

$$
\mu(\mathcal{A})=\min \{\mu ; \mu \text { is Pareto } Z \text {-eigenvalue of } \mathcal{A}\}=\min _{\substack{\mathbf{y} \geq 0 \\\|\mathbf{y}\|_{2}=1}} \mathcal{A} \mathbf{y}^{m}>0
$$


Similarly, we also may assume that $\mathbf{q}$ is not nonnegative, then $\mathbf{x} \neq \mathbf{0}$, and so

$$
\frac{\mathcal{A} \mathbf{x}^{m}}{\|\mathbf{x}\|_{2}^{m}}=\mathcal{A}\left(\frac{\mathbf{x}}{\|\mathbf{x}\|_{2}}\right)^{m} \geq \mu(\mathcal{A})>0 .
$$

Thus, we have

$$
0<\|\mathbf{x}\|_{2}^{m} \mu(\mathcal{A}) \leq \mathcal{A} \mathbf{x}^{m}=\mathbf{x}^{\top}(-\mathbf{q}) \leq \mathbf{x}^{\top}(-\mathbf{q})_{+} \leq\|\mathbf{x}\|_{2}\left\|(-\mathbf{q})_{+}\right\|_{2} .
$$

The desired conclusion follows.

We now introduce a quantity for a strictly semi-positive tensor $\mathcal{A}$.

$$
\beta(\mathcal{A}):=\min _{\substack{\mathbf{x} \geq 0 \\ \mathbf{x} \| \infty=1}} \max _{i \in I_{n}} x_{i}\left(\mathcal{A} \mathbf{x}^{m-1}\right)_{i},
$$

where $\|\mathbf{x}\|_{\infty}:=\max \left\{\left|x_{i}\right| ; i \in I_{n}\right\}$. It follows from the definition of strictly semi-positive tensor that $\beta(\mathcal{A})>0$. Then the following equation is well defined in Theorem 3.8,

Theorem 3.8. Let $\mathcal{A}=\left(a_{i_{1} \cdots i_{m}}\right) \in T_{m, n}$ be strictly semi-positive. If $\mathbf{x}$ is a solution of the $\operatorname{TCP}(\mathbf{q}, \mathcal{A})$, then

$$
\|\mathbf{x}\|_{\infty}^{m-1} \leq \frac{\left\|(-\mathbf{q})_{+}\right\|_{\infty}}{\beta(\mathcal{A})} .
$$

Proof Suppose that $\mathbf{q} \geq \mathbf{0}$. Then $\mathbf{x}=\mathbf{0}$ by Lemma 3.1, the conclusion is obvious. So we may assume that $\mathbf{q}$ is not nonnegative, similarly to the proof technique of Theorem 3.6, we have $\mathbf{0} \leq \mathbf{x} \neq \mathbf{0}$. It follows from the definition of $\beta(\mathcal{A})$ and (2.4) that

$$
\begin{aligned}
0<\|\mathbf{x}\|_{\infty}^{m} \beta(\mathcal{A}) & \leq \max _{i \in I_{n}} x_{i}\left(\mathcal{A} \mathbf{x}^{m-1}\right)_{i}=\max _{i \in I_{n}} x_{i}(-q)_{i} \\
& \leq \max _{i \in I_{n}} x_{i}\left((-q)_{+}\right)_{i} \leq\|x\|_{\infty}\left\|(-q)_{+}\right\|_{\infty} .
\end{aligned}
$$

The desired conclusion follows.

It is well known that if a nonlinear complementarity problem with pseudo-monotone and continuous function $F$ has a strictly feasible point $x^{*}$ (i.e., $\mathbf{x}^{*} \geq 0, F\left(\mathbf{x}^{*}\right)>0$ ), then it has a solution ([3, Theorem 2.3.11]). A function $F$ from $\mathbb{R}_{+}^{n}$ into itself is called pseudo-monotone iff for all vectors $\mathbf{x}, \mathbf{y} \in \mathbb{R}_{+}^{n}$,

$$
(\mathbf{x}-\mathbf{y})^{\top} F(\mathbf{y}) \geq 0 \Rightarrow(\mathbf{x}-\mathbf{y})^{\top} F(\mathbf{x}) \geq 0 .
$$

Now we give an example to certify the function $F$ deduced by a strictly semi-positive tensor is not pseudo-monotone. However, the corresponding nonlinear complementarity problem has a solution by Lemma 2.1. 
Example 3.1. Let $\mathcal{A}=\left(a_{i_{1} i_{2} i_{3}}\right) \in T_{3,2}$ and $a_{111}=1, a_{122}=1, a_{211}=1, a_{221}=-2, a_{222}=1$ and all other $a_{i_{1} i_{2} i_{3}}=0$. Then

$$
\mathcal{A} \mathbf{x}^{2}=\left(\begin{array}{l}
x_{1}^{2}+x_{2}^{2} \\
x_{1}^{2}-2 x_{1} x_{2}+x_{2}^{2}
\end{array}\right) .
$$

Clearly, $\mathcal{A}$ is strictly semi-positive, and so it is a Q-tensor.

Let $F(\mathbf{x})=\mathcal{A} \mathbf{x}^{2}+\mathbf{q}$ for $\mathbf{q}=\left(-\frac{3}{2},-\frac{1}{2}\right)^{\top}$. Then $F$ is not pseudo-monotone. In fact,

$$
F(\mathbf{x})=\mathcal{A} \mathbf{x}^{2}+\mathbf{q}=\left(\begin{array}{c}
x_{1}^{2}+x_{2}^{2}-\frac{3}{2} \\
\left(x_{1}^{2}-x_{2}\right)^{2}-\frac{1}{2}
\end{array}\right) .
$$

Take $\mathbf{x}=(1,0)^{\top}$ and $\mathbf{y}=(1,1)^{\top}$. Then

$$
\mathbf{x}-\mathbf{y}=\left(\begin{array}{r}
0 \\
-1
\end{array}\right), F(\mathbf{x})=\left(\begin{array}{r}
-\frac{1}{2} \\
\frac{1}{2}
\end{array}\right) \text { and } F(\mathbf{y})=\left(\begin{array}{r}
\frac{1}{2} \\
-\frac{1}{2}
\end{array}\right)
$$

Clearly, we have

$$
(\mathbf{x}-\mathbf{y})^{\top} F(\mathbf{y})=0 \times \frac{1}{2}-1 \times\left(-\frac{1}{2}\right)>0 .
$$

However,

$$
(\mathbf{x}-\mathbf{y})^{\top} F(\mathbf{x})=0 \times\left(-\frac{1}{2}\right)-1 \times \frac{1}{2}<0,
$$

and hence $F$ is not pseudo-monotone.

\section{Perspectives}

There are more research topics on solution of the tensor complementarity problem for further research.

We show the global upper bounds for solution of $\operatorname{TCP}(\mathcal{A}, \mathbf{q})$ with strictly semi-positive (symmetric) tensor $\mathcal{A}$. Then we have the following questions for further research.

(i) Do it has a positive lower bound?

(ii) Are the above upper bounds is the smallest?

(iii) For some other structured tensors such as Z-tensors, B-tensors, H-tensors, M-tensors and so on, whether do they have similar upper bounds or not?

We define a quantity $\beta(\mathcal{A})$ for a strictly semi-positive tensor $\mathcal{A}$. Then does the quantity have a global (or local) upper bound? 


\section{Conclusions}

In this paper, We discuss the equivalent relationships between the feasible solution of the tensor complementarity problem and S-tensor, the boundedness of solution of the tensor complementarity problem and $\mathrm{R}_{0}$-tensor. By means of two constants, keep in close contact with the smallest Pareto $H-(Z-)$ eigenvalue, we proved the global upper bounds for solution of the tensor complementarity problem with a strictly semi-positive and symmetric tensor. A new quantity is introduced for a strictly semi-positive tensor. For a strictly semi-positive tensor (not symmetric), the global upper bounds for such a tensor complementarity problem is proved by means of such a new quantity.

\section{Acknowledgment}

The authors would like to thank the editors and anonymous referees for their valuable suggestions which helped us to improve this manuscript.

\section{References}

1. Ferris, M. C., Pang, J. S.: Engineering and Economic Applications of Complementarity Problems. SIAM Review 39(4), 669-713 (1997)

2. Harker, P. T., Pang, J. S.: Finite-dimensional variational inequality and nonlinear complementarity problems: A survey of theory, algorithms and applications. Math. Program. 48(1-3), 161-220 (1990)

3. Han, J.Y., Xiu, N.H., Qi, H.D.: Nonlinear complementary Theory and Algorithm. Shanghai Science and Technology Press, Shanghai. (2006) (in Chinese)

4. Facchinei, F., Pang, J.S.: Finite-Dimensional Variational Inequalities and Complementarity Problems: Volume I, Springer-Verlag, New York Inc. (2003)

5. Cottle, R.W., Pang, J.S., Stone, R.E.: The Linear Complementarity Problem. Academic Press, Boston (1992)

6. Cottle, R.W., Randow, R.V.: Complementary pivot theory of mathematical programming. Linear Algebra Appl. 1, 103-125 (1968)

7. Eaves, B.C.: The linear complementarity problem. Management Science 17, 621-634 (1971)

8. Karamardian, S.: The complementarity problem. Math. Program. 2, 107-129 (1972) 
9. Pang, J.S.: On Q-matrices. Math. Program. 17, 243-247 (1979)

10. Pang, J.S.: A unification of two classes of Q-matrices. Math. Program. 20, 348-352 (1981)

11. Seetharama Gowda, M.: On Q-matrices. Math. Program. 49, 139-141 (1990)

12. Cottle, R.W.: Completely Q-matrices. Math. Program. 19, 347-351 (1980)

13. Song, Y., Qi, L.: Properties of Tensor Complementarity Problem and Some Classes of Structured Tensors. arXiv:1412.0113v1 (2014)

14. Che, M., Qi, L., Wei, Y.: Positive definite tensors to nonlinear complementarity problems. J Optim Theory Appl. 168 475-487 (2016) arXiv.1501.02546v1 (2015)

15. Luo, Z., Qi, L., Xiu, N.: The sparsest solutions to $Z$-tensor complementarity problems. Optimization Letters, DOI 10.1007/s11590-016-1013-9 (2015) arXiv: 1505.00993 (2015)

16. Song, Y., Qi, L.: Tensor complementarity problem and semi-positive tensors, J Optim Theory Appl. 169(3) 1069-1078 (2016) arXiv:1502.02209 (2015)

17. Qi, L.: Eigenvalues of a real supersymmetric tensor. J. Symbolic Comput. 40, 1302-1324 (2005)

18. Zhang, L., Qi, L., Zhou, G.: M-tensors and some applications. SIAM J. Matrix Anal. Appl. 35(2), 437-452 (2014)

19. Ding, W., Qi, L., Wei, Y.: M-tensors and nonsingular M-tensors. Linear Algebra Appl. 439, 3264-3278 (2013)

20. Song, Y., Qi, L.: Properties of Some Classes of Structured Tensors. J. Optim. Theory Appl. 165(3), 854-873 (2015)

21. Qi, L., Song, Y.: An even order symmetric B tensor is positive definite. Linear Algebra Appl. 457, 303-312 (2014)

22. Song, Y., Qi, L.: Infinite and finite dimensional Hilbert tensors. Linear Algebra Appl. 451, 1-14 (2014)

23. Song, Y., Qi, L.: Spectral properties of positively homogeneous operators induced by higher order tensors. SIAM J. Matrix Anal. Appl. 34(4), 1581-1595 (2013)

24. Qi, L.: Symmetric Nonnegative Tensors and Copositive Tensors. Linear Algebra Appl. 439(1), 228-238 (2013) 
25. Song, Y., Qi, L.: Eigenvalue analysis of constrained minimization problem for homogeneous polynomial, J Global Optim. 64(3), 563-575 (2016) arXiv:1302.6085 (2013)

26. Ling, C., He, H., Qi, L.: On the cone eigenvalue complementarity problem for higherorder tensors. Comput. Optim. Appl. 63, 143-168 (2016)

27. Chen, Z., Yang, Q., Ye, L.: Generalized Eigenvalue Complementarity Problem for Tensors. arXiv:1505.02494 (2015) 